\title{
Alterstice
}

Revue internationale de la recherche interculturelle

International Journal of Intercultural Research

Revista International de la Investigacion Intercultural

\section{Nature de la violence conjugale et familiale et obstacles à l'utilisation des services pour les femmes indiennes immigrantes [synthèse en français]}

\author{
Erminia Colucci, Manjula O’Connor, Karen Field, Alice Baroni, Reima Pryor et \\ Harry Minas
}

Volume 3, numéro 2, 2013

Violence conjugale et diversité culturelle

URI : https://id.erudit.org/iderudit/1077517ar

DOI : https://doi.org/10.7202/1077517ar

Aller au sommaire du numéro

Éditeur(s)

Alterstice

ISSN

1923-919X (numérique)

Découvrir la revue

Citer ce document

Colucci, E., O’Connor, M., Field, K., Baroni, A., Pryor, R. \& Minas, H. (2013).

Nature de la violence conjugale et familiale et obstacles à l'utilisation des

services pour les femmes indiennes immigrantes [synthèse en français].

Alterstice, 3(2), i-iv. https://doi.org/10.7202/1077517ar 


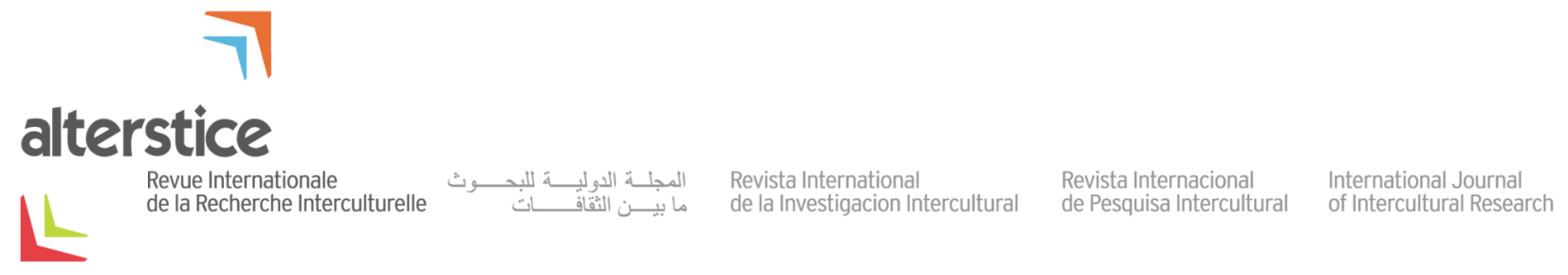

ARTICLE THÉMATIQUE

\title{
Nature de la violence conjugale et familiale et obstacles à l'utilisation des services pour les femmes indiennes immigrantes [synthèse en français*]
}

\author{
Erminia Colucci ${ }^{1}$, Manjula $\mathrm{O}^{\prime}$ Connor $^{1}$, Karen Field $^{1}$, Alice Baroni ${ }^{1}$, Reima Pryor $^{1}$ et Harry Minas ${ }^{1}$
}

\author{
Rattachement des auteurs \\ ${ }^{1}$ Centre for International Mental Health, School of Population and Global Health, University of Melbourne, \\ Melbourne, Australie.
}

\section{Correspondance}

ecolucci@unimelb.edu.au

\section{Mots clés}

migration; violence conjugale; violence familiale; violence envers les femmes; Indiennes; culture; recherche à composante artistique; théâtre forum; ethnographie de la performance artistique; utilisation des services; accès aux services; prévention de la violence.

\section{Pour citer cet article :}

Colucci, E., O’Connor, M., Field, K., Baroni, A., Pryor, R. et Minas, H. (2013). Nature de la violence conjugale et familiale et obstacles à l'utilisation des services pour les femmes indiennes immigrantes [synthèse en français*]. Alterstice, 3(2), v-viii.

*L'article complet en anglais est disponible sur le site d'Alterstice.

\section{Contexte}

La violence envers les femmes, notamment la violence conjugale, est un phénomène universel qui perdure dans tous les pays et toutes les sociétés du monde (OMS, 2005) et qui touche toutes les communautés, sans distinction de race, de genre, de classe sociale, de religion, d'origine culturelle ou d'ethnicité (Bannenberg et Tossner, 2003). Cependant, il existe des variations d'une nation et d'une culture à l'autre. En Australie, plus du tiers des femmes (40\%) de quinze ans ou plus sont victimes de violence physique ou sexuelle (VicHealth, 2011).

Des chercheurs et des fournisseurs de services ont mis en évidence le niveau particulièrement élevé de la violence subie par les femmes issues de communautés immigrantes ou réfugiées (Bonar et Roberts, 2006; Choi, Cheung et Cheung, 2012). Par ailleurs, ces femmes font face à davantage d'obstacles que les femmes en général pour réussir à chercher de l'aide (Intouch, 2010; Orloff et Little, 1999; Raj et Silverman, 2002). 
Le théâtre forum est un outil efficace qui se sert du théâtre pour renforcer la compréhension dans la communauté des problèmes difficiles à traiter comme l'est la violence conjugale. En alliant l'ethnographie et le théâtre, notre objectif consistait à définir les principaux problèmes, défis et besoins des familles indiennes immigrantes lorsqu'elles tentent d'accéder et de recourir à des services susceptibles de leur venir en aide dans des situations de violence conjugale ou familiale.

\section{Méthode}

Cette expérience ethnographique s'est déroulée en trois étapes : séances d'information et groupes de discussion, ateliers de théâtre et pièces de théâtre jouées devant la communauté. Soixante-douze Indiennes ont pris part à quatre groupes de discussion, quatorze Indiennes ont participé à une partie ou à l'ensemble des six ateliers d'une journée (huit heures) répartis sur une période de quatre semaines et cent quatorze femmes, majoritairement des Indiennes, ont assisté à cinq représentations communautaires.

\section{Résultats}

Les participantes ont révélé la présence de plusieurs formes de violence conjugale dans leur communauté et discuté des comportements qui nourrissent de telles pratiques ainsi que des difficultés à recevoir de l'aide et à accéder aux services. Les observations clés ont été réparties dans les deux domaines d'enquête principaux suivants : nature de la violence conjugale et obstacles à la recherche d'aide.

Bien que les participantes aient évoqué les mêmes types de violence conjugale que les femmes non immigrantes (violence physique, sexuelle, financière, sociale, spirituelle et émotionnelle), certaines formes spécifiques de violence émotionnelle ou psychologique ont été mises en lumière et présentées comme les formes les pires de violence. L'inégalité entre les hommes et les femmes combinée au peu de liberté dont les Indiennes disposent pour prendre des décisions a été perçue comme générant un contexte culturel propice à la violence. Les participants ont mentionné des menaces de déportation et d'annulations des visas d'immigration. De nombreuses femmes ont décrit le "silence " qu'elles étaient censées garder et l'obligation d'écouter les autres. Les participantes ont également décrit des formes de violence sociale telles que la peur d'être jugée par la communauté ou le contrôle de leurs interactions sociales par leur mari. Parmi les formes de violence financière figuraient le refus d'accorder l'indépendance financière aux femmes ou encore le contrôle de leurs revenus par autrui ainsi que des attentes élevées en matière de dot.

La pression de devoir soutenir financièrement les membres de la famille restés en Inde a également été définie comme un facteur de stress, de conflit, voire de violence. Les femmes ont évoqué des formes de violence physique et sexuelle et ont éprouvé beaucoup de difficulté à remettre en question la violence sexuelle dans un contexte conjugal. La violence spirituelle ou religieuse se manifestait sous la forme d'interdiction pour les femmes d'aller au temple, de prier ou de faire des offrandes. Bien que certaines participantes aient bien identifié les comportements relevant de la violence conjugale, d'autres parvenaient difficilement à distinguer les pratiques et les comportements considérés comme culturellement "normaux » et acceptables dans la communauté indienne de ceux susceptibles d'être classés comme forme de violence conjugale.

Si les " agresseurs » étaient habituellement les maris, les participants ont parfois désigné des parents du conjoint (frères, sœurs, père ou mère). Des participantes ont précisé que ces " agresseurs » ne vivaient pas nécessairement en Australie.

Les participantes ont décrit les facteurs de stress qui contribuaient aux conflits et à la violence, notamment la déception et l'échec vécus par les immigrants pleins d'espoir échouant à obtenir le travail ou le revenu attendus.

Le manque de soutien social a également été présenté comme contribuant à la violence, soit parce qu'il peut générer des conflits qui conduisent à la violence, soit parce qu'il favorise l'irresponsabilité de l'homme et la vulnérabilité de la femme à la violence. Finalement, les changements d'attitude et de comportement de la femme à la suite de l'immigration peuvent ne pas être acceptés par l'homme et l'amener à se montrer violent envers sa femme. 
Au cours du projet, il est apparu clairement que les obstacles empêchant les immigrantes indiennes de recevoir de l'aide se divisaient en deux catégories : la première est liée aux obstacles généraux à la dénonciation de la violence conjugale et à l'accès au soutien social informel et la seconde est liée aux obstacles particuliers à l'accès à l'aide professionnelle. Des solutions stratégiques ont été proposées pour donner aux membres de la communauté le pouvoir de "briser le silence ", aider les femmes à chercher de l'assistance et leur présenter " de bons modèles d'identification de femmes indiennes » pouvant bousculer les habitudes de silence et de soumission.

En plus des problèmes culturels décrits ci-dessus, les participantes ont mentionné le manque de sensibilisation à la violence et de connaissance des lois et du droit. Elles ont souligné l'importance d'informer adéquatement les immigrantes, particulièrement au moment de leur arrivée, de leurs droits et des lois en vigueur dans leur pays d'accueil.

Les services sociaux ont rarement fait partie du "discours » des participantes sur la violence conjugale et les femmes semblaient préférer chercher de l'aide auprès de la famille immédiate et de la communauté en général que de l'aide professionnelle. La préférence marquée pour un professionnel d'origine indienne, en raison d'une compréhension commune de la culture, de la facilité à nouer des relations et de la connaissance de la langue est perçue comme un obstacle à l'accès aux services.

Les participantes avaient le sentiment que les services « allaient trop vite, les poussaient à franchir des étapes trop grandes " alors que les femmes " ont besoin d'avancer à petits pas », en particulier quand la solution proposée pour remédier à une situation de violence conjugale consistait à " quitter le mari », qui n'est pas « la façon indienne de faire les choses ". D'autres formes d'aide ont été accueillies positivement: les programmes d'aide sociale et de prévention de la violence, les activités visant à renforcer l'autonomie des femmes, les lignes d’aide téléphoniques et les groupes communautaires comme les groupes d'entraide.

Le manque d'information à propos des services (nature des services et lieux de leur prestation) et de la manière d'y accéder représente également un obstacle important à l'accès aux services et à l'aide professionnelle. Pour ce qui est des procédures bureaucratiques, les inquiétudes portaient principalement sur les formulaires d'évaluation et de consentement considérés avec beaucoup de scepticisme et de méfiance, par crainte des conséquences que pourrait entraîner la divulgation de renseignements personnels sur leur statut d'immigration.

\section{Conclusion}

Cette étude corrobore les éléments de preuves selon lesquels les femmes marginalisées par l'âge, la culture, l'ethnicité, l'identité sexuelle et le statut d'immigration (type de visa) sont davantage susceptibles d'être victimes de violence et de ne pas avoir accès aux ressources nécessaires pour remédier à une situation de violence (Poljski, 2011), ce qui semble être le cas des femmes immigrantes d'origine indienne. Les participantes ont éprouvé de la difficulté à distinguer les pratiques et les comportements culturellement "normaux " et acceptables dans la communauté indienne des comportements qui pourraient, de fait, être considérés comme des formes de violence conjugale. Les participantes ont réfléchi sur les facteurs sociaux qui contribuent à la violence et analysé la dynamique de la violence conjugale dans leur communauté. Les fournisseurs de services doivent comprendre la dynamique complexe de la violence conjugale dans les familles indiennes, ainsi que la dynamique familiale en général, et pouvoir agir sur celle-ci.

Cette étude a mis en lumière plusieurs facteurs culturels et sociaux qui nuisent à l'accès aux services. Le lien entre le statut d'immigration, la vulnérabilité à la violence et l'utilisation des services montre la nécessité d'apporter des changements au système et d'informer les nouveaux arrivants. De premières recommandations sont proposées, mais il faudrait approfondir les recherches pour mettre sur pied des stratégies d'atténuation des facteurs de risque pour les femmes indiennes. En plus de nous avoir permis de mieux comprendre la nature de la violence conjugale et les obstacles culturels à la recherche d'aide, ce projet a montré que les spectacles ont également contribué à changer l'attitude de la communauté indienne envers les femmes et relativement à la violence contre les femmes et à ouvrir la discussion sur un sujet souvent tu. 


\section{Références*}

Bannenberg, B. et Rossner, D. (2003). New developments in restorative justice to handle family violence. Dans E. Weitekamp et H. Kerner (dir.), Restorative justice in context: International practice and directions (p. 5179). Uffculme, Royaume-Uni : Willan Publishing.

Bonar, M. et Roberts, D. (2006). A review of literature relating to family and domestic violence in communities in Australia. Western Australia, Australie : Government of Western Australia, Family and Domestic Violence Unit.

Choi, S. , Cheung, Y. et Cheung, A. (2012). Social isolation and spousal violence: Comparing female marriage migrants with local women. Journal of marriage and family, 74, 444-461.

Intouch. (2010). Barriers to the justice system faced by CALD women experiencing family violence. Melbourne, Australie : InTouch Multicultural Centre Against Family Violence.

Orloff, L. et Little, R. (1999). Somewhere to turn: Making domestic violence services accessible to battered immigrant women. A “How to” manual battered women's advocates and service providers. États-Unis : Ayuda Inc.

Poljski, C. (2011). On her way: Primary prevention of violence against immigrant and refugee women in Australia. Melbourne, Australie : Multicultural Centre for Women's Health.

http://www.vichealth.vic.gov.au/en/Publications/Freedom-from-violence/On-Her-Way.aspx

Raj, A. et Silverman, J. (2002). Violence against immigrant women: The roles of culture, context, and legal immigrant status on intimate partner violence. Violence against women, 8(3), 367-398.

VicHealth. (2011). Preventing violence against women in Australia. Melbourne, Australie : Victorian Health Promotion Foundation.

OMS (2005). Étude multipays de l'OMS sur la santé des femmes et la violence domestique à l'égard des femmes. Rapport succinct : premiers résultats concernant la prévalence, les effets sur la santé et les réactions des femmes. Genève, Suisse : Organisation mondiale de la santé. http://www.who.int/reproductivehealth/publications/violence/9241593512/fr/

*Voir l'article en anglais pour la bibliographie d'ensemble. 